\title{
Health service experiences and preferences of frail home care clients and their family and friend caregivers during the COVID-19 pandemic
}

\author{
Lori E. Weeks ${ }^{1^{*}}$ (1), Sue Nesto ${ }^{2}$, Bradley Hiebert ${ }^{3}$, Grace Warner ${ }^{2}$, Wendy Luciano ${ }^{2}$, Kathleen Ledoux ${ }^{3}$ and \\ Lorie Donelle ${ }^{3}$
}

\begin{abstract}
Objective: The COVID-19 pandemic has brought about a major upheaval in the lives of older adults and their family/ friend caregivers, including those utilizing home care services. In this article, we focus on results from a qualitative component added to a pragmatic randomized controlled trial that focuses on the experiences of our study participants during COVID-19. A total of 29 participants responded to the COVID-19 related questions focused on their health services experiences and preferences from March-June 2020 including 10 home care clients and 19 family/ friend caregivers in the provinces of Ontario and Nova Scotia, Canada.

Results: Many participants were affected drastically by the elimination or reduction of access to services, highlighting the vulnerability of home care clients and their caregivers during COVID-19. This took an emotional toll on home care clients and increased the need for family/friend caregiver support. While many participants expressed reduced desire to utilize residential long-term care homes, some caregivers found that passive remote monitoring technology was particularly useful within the COVID-19 context. Our results provide important insights into the ways the older adults and their caregivers have been affected during the COVID-19 context and how to better support them in the future.
\end{abstract}

Keywords: COVID-19, Remote monitoring, Home care, Older adults, Frailty

\section{Introduction}

The COVID-19 pandemic has brought about a major upheaval in the lives of older adults and their family/ friend caregivers [1] including highlighting the vulnerability of older adults living in long-term care (LTC) homes [2]. Many community-dwelling frail older adults are also at a high risk of morbidity and mortality and many have experienced a loss of services deemed nonessential that can contribute to feeling lonely and

*Correspondence: lori.weeks@dal.ca

1 School of Nursing, Dalhousie University, Halifax, NS B3H 4R2, Canada

Full list of author information is available at the end of the article abandoned [3]. We know little about the experiences of community-dwelling frail older adults and their caregivers during the pandemic.

Our research team is engaged in a pragmatic randomized controlled trial study, "Caring Near and Far," that is examining the effectiveness of passive remote monitoring technologies (RMT) for frail older adults receiving home care services defined as being likely to be admitted to a LTC home within the next 12 months. Passive RMT uses sensors that do not require any action by the individual for the system to work, as opposed to active RMT that requires individual participation, such as pushing a button. Information gathered from the sensors (e.g., 
motion sensors, cameras) is transmitted to a caregiver to alert them to a possible incident (e.g., a fall, wandering). Quantitative and qualitative data was collected from dyads of home care clients and their family/friend caregivers at three time points in the provinces of Ontario $(\mathrm{ON})$ and Nova Scotia (NS), Canada. For further details on our study methodology, please see the published protocol [4].

The purpose of this study is to identify the health service experiences and preferences of frail home care clients and their family and friend caregivers during the COVID-19 pandemic. We address the following research questions:

1. How has the utilization of home and communitybased services been affected during COVID-19 for frail older adults and their caregivers?

2. What is the impact of changed access to services during COVID-19?

3. How has COVID-19 affected decisions and preferences about LTC placement?

4. How has the utilization of passive RMT been affected by COVID-19?

\section{Main text \\ Methods}

We invited home care clients and their caregivers to respond to our COVID-19 related questions either during regularly scheduled interviews as part of our larger pragmatic randomized controlled trial or during a separate interview.

\section{Data collection}

Research coordinators and research assistants conducted the interviews by telephone. Open-ended questions added to this study focused on understanding how our study participants have been affected by COVID-19, including their utilization and preferences of health services. These questions added approximately 10 to $30 \mathrm{~min}$ of interview time.

\section{Setting and recruitment}

A total of 29 participants volunteered to respond to the COVID-19 related questions from March-June 2020 including 10 home care clients and 19 caregivers. Home care clients' dependence on their family/friend caregivers was assessed by determining their risk of requiring higher levels of care according to the Mini Mental-State Exam [5] (to determine cognitive functioning) and the HARP Independent Activities of Daily Living assessment [6] (to determine physical functioning). These assessments indicated two home care clients were at elevated risk for requiring higher levels of care due to both cognitive decline and physical decline, six were at elevated risk due to physical decline, and two exhibited neither cognitive nor physical decline to the degree that would require elevated levels of care. The participants were located in ON $(n=15)$ and NS $(n=14)$. A total of 14 people interviewed were in the study intervention arm and 15 received usual care.

\section{Data analysis}

The interviews were transcribed verbatim and field notes were added by the interviewers. Through adapting a framework method [7] to guide our data analysis process, we deductively organized the qualitative results according to our 4 questions and then inductively identified themes with each question utilizing thematic analysis [8]. To ensure auditability, members of our research team involved in interviewing and managing qualitative data provided insights into important findings emerging from the interviews.

\section{Results}

See Table 1.

Table 1 Questions and themes

\begin{tabular}{|c|c|}
\hline Questions & Themes \\
\hline \multirow{2}{*}{$\begin{array}{l}\text { 1. How has the utilization of home and community-based services been affected during COVID-19 } \\
\text { for frail older adults and their caregivers? }\end{array}$} & Service cancellation \\
\hline & $\begin{array}{l}\text { Modified access with infection control practices } \\
\text { implemented }\end{array}$ \\
\hline \multirow[t]{2}{*}{ 2. What is the impact of changed access to services during COVID-19 } & Emotional and physical toll on home care clients \\
\hline & Increased need for family caregiver support \\
\hline \multirow[t]{2}{*}{ 3. How has COVID-19 affected decisions and preferences about LTC placement? } & Less likely to want to go to LTC \\
\hline & I will go if I have to \\
\hline \multirow[t]{3}{*}{ 4. How has the utilization of passive RMT been affected by COVID-19? } & Less utilization \\
\hline & No impact on utilization \\
\hline & Increased utilization \\
\hline
\end{tabular}


How has the utilization of home care services been affected during COVID-19 for frail older adults and their caregivers? Service cancellation For many, the impact of COVID-19 on home care services resulted in the client or caregiver cancelling services for many reasons including: different home care workers being assigned to one client; home care workers also working in nursing homes; or having a homecare worker who tested positive for COVID-19. For some, canceling home care services was a mutual decision. "They ended up cancelling themselves. But we had talked to them beforehand.... I don't think that the homecare will come back until they're satisfied that things are safe" (NS client 212,155).

Other services in the home, such as respite were affected during COVID-19. "So we were actually the best part of two months with no help." (NS caregiver 222,154).

Modified access with infection control practices implemented Some participants continued to receive home care services during the pandemic. "I respect that they put masks on and that they wear gloves too" (ON client 113,138). Home care agencies conducted screening prior to coming into a client's home. One participant established their own protocols to screen home-care workers coming into their home. Many participants expressed concerns about multiple home care workers coming into the home. "We have so many people coming in the house, we make sure everybody's got masks on and they're following protocol." (ON caregiver 123,181). Some participants had access to physicians who provided house calls or appointments over the phone. Several participants spoke about continued access to pharmacy services and safe ways to pick up orders or prescription delivery.

\section{What is the impact of changed access to services during COVID-19?}

Emotional and physical toll on home care clients Some participants expressed that they missed their care workers and found it lonely having fewer or no home care providers in their homes. In addition, many older adult clients reported having to do more care for themselves, such as bathing, and struggled to care for themselves safely. "He had personal care that came at least once a week, and he doesn't have that anymore. And I think he kind of misses that because ... when he gets a bath, he's more comfortable knowing that there's someone there in case he falls" (NS caregiver 122,137).

Increased need for family caregiver support The impact of reduced services often resulted in family members providing more supports. Providing additional support was very challenging for many caregivers. One caregiver reported a two-month gap in other supports in the home. Some family members chose to live with their relative receiving home care. "Our son is now staying with us. And he does work, but he comes in the basement door, strips, showers, puts his clothes in the laundry, and then upstairs. So we feel very safe with him" (NS caregiver 122,152). Caregivers who lived with the home care client in addition to working from home faced particular challenges: "Where if we were generally working, she would be taking her own medications in certain doses, preparing her lunch herself. So, it's changed certainly that we are here so she relies on us more as well" (ON caregiver 123,165).

\section{How has COVID-19 affected decisions and preferences about LTC placement?}

Less likely to want to go to LTC The majority of home care clients did not want to go into LTC during the pandemic. "The deaths that are being happening in these long-term care nursing homes. I think if a person is being sent to a long-term care, it's suicide" (NS client 212,155). Most caregivers stated they would not place their family members there during the pandemic. "I'm just glad she wasn't in a facility because I felt I would have taken her out" (NS caregiver 122,107). One caregiver wanted enhanced regulation before she would consider LTC for her husband.

I will go if I have to Conversely, some participants indicated that they would consider LTC placement if their health declined. "I would probably go if I was in that condition of not looking after myself" (NS client 112,152). They recognized that not all LTC homes experienced outbreaks of COVID-19. Another participant had experience working in LTC and was not concerned if moving to one became a necessity. "I don't really have any concerns. I worked in them, so I know the system" (ON client 113,138).

\section{How has the utilization of passive RMT been affected by COVID-19?}

Less utilization In some cases, passive RMT was used less due to family members being more physically available during COVID-19. "I'm not able to use it as much as I did because I'm not going out...prior to COVID-19, I would use the monitor, the camera more because I was out (NS caregiver 222,155).

No impacton utilization In some instances, there was no impact of COVID-19 on the use of the technology. As the system is designed to work passively, many clients think very little about the technology in their homes. "With the COVID virus or without the COVID virus, it is basically the same thing because you're able to do it without being in contact" (NS Caregiver 222,117). 
Increased utilization Some caregivers found that passive RMT was more helpful during COVID-19 to ensure their loved-one was safe and secure. Caregivers who did not live with the home care client were comforted by being able to use the cameras to check on their family member while not having to be physically present and potentially spreading COVID-19. Some caregivers used the camera to monitor the number of different people coming into the home to provide personal care. Another caregiver preferred to monitor her mother using the technology rather than have multiple different home care workers coming into the home. The acceptance of various forms of technologies increased during the pandemic, especially if it supported being able to remain at home.

\section{Discussion}

Some caregivers found that passive RMT technology was particularly useful within the COVID-19 pandemic context and some clients benefited from knowing that someone was monitoring them. These functions of passive RMTs may become more valued during the pandemic as monitoring can be done without physical contact. There may also be new applications of RMT for home care agencies who could monitor clients without going into the home.

It is imperative to support family and friend caregivers who may need to provide additional care during the COVID-19 pandemic out of necessity. While identifying the need to support caregivers of older adults is well-established $[9,10]$, this need is more intensified in the pandemic context. Creative ways to support caregivers are needed, such as supports that can be provided remotely (e.g., online support groups).

A key concern raised by our participants was having multiple home care providers coming into their homes. The issue of care staff working across multiple LTC homes has been clearly identified to mitigate the spread of COVID-19 [11]. Prior to the COVID-19 context, the lack of consistent home care providers was identified as a concern about quality of care $[12,13]$. The current pandemic may provide additional impetus for improving various aspects that could contribute to staff retention such as improving compensation, education and training, and working conditions $[14,15]$.

In addition to changes in services, the pandemic created a situation in which many people were even less open to considering moving to a LTC home. Most older adults prefer to live as independently as possible in their own homes in the community [16]. Our results indicate that this may be amplified during the COVID19 pandemic context, and there may be even greater need to expedite the development of innovative housing and care options to meet the needs of frail older adults who do not wish to move to a traditional LTC home.

In conclusion, there was clearly a diversity of experiences of the participants in our study within the context of the COVID-19 pandemic. While we expected that many community-dwelling older adults would be affected by a loss or modification of home care services [3], in this study we provided additional insights into the impact of COVID-19 on the lives of home care clients and their caregivers. Many were affected drastically by the elimination or reduction of access to services. Our results highlight the vulnerability of home care clients and their caregivers during the pandemic.

\section{Limitations}

There are limitations of the utility of passive RMT within the context of the COVID-19 pandemic as it does not address the social isolation that many older adults may be experiencing. Also, not all home care clients have equal access to high-speed internet which could create disparities for those living in urban versus rural or remote locations. We collected data in two Canadian provinces. Our results can not be transferred to other jurisdictions, especially where different home care practices have been employed during the pandemic (e.g., different ways that services have been modified).

\section{Abbreviations}

LTC: Long-term care; NS: Nova Scotia; ON: Ontario; RMT: Remote monitoring technology.

\section{Acknowledgements}

We thank the entire Caring Near and Far research team, study participants, and our advisory groups for their contributions to this research.

\section{Authors' contributions}

LD, GW, KL, SN, and LEW made substantial contributions to the conception and design of this work. SN, WL, and $\mathrm{BH}$ contributed to the acquisition of data. All authors contributed to data analysis and interpretation. LEW drafted the manuscript, and all authors were involved in the revision of the manuscript. All authors approved the submitted manuscript and have agreed to be personally accountable for their own contributions and to the accuracy and integrity of the research. All authors read and approved the final manuscript.

\section{Funding}

Funding for this was research was provided by the Canadian Institutes of Health Research Operating Grant through the eHealth Innovation Partnership Program-Seniors with Complex Care Needs (EH2-143561). The funder had no role in the design of the study, data collection, data analysis, or interpretation of the results.

\section{Availability of data and materials}

The data analysed during this study are not publicly available as the qualitative data may be identifiable. Questions about access to deidentified data should be addressed to the corresponding author. 


\section{Declarations}

\section{Ethics approval and consent to participate}

This research was approved by the Nova Scotia Health Authority Research Ethics Board (\#1022203) and the Western Research Ethics Board (\#108406) in Ontario. Participants signed a research consent form.

\section{Consent for publication}

Individual participants provided consent for deidentified quotes from their interviews to be included in any dissemination of the results.

\section{Competing interests}

The authors declare that they have no competing interests.

\section{Author details}

'School of Nursing, Dalhousie University, Halifax, NS B3H 4R2, Canada. ${ }^{2}$ School of Occupational Therapy, Dalhousie University, Halifax, NS B3H 4R2, Canada.

${ }^{3}$ Arthur Labatt School of Nursing, Western University, London, ON, Canada.

Received: 22 December 2020 Accepted: 6 July 2021

Published online: 14 July 2021

\section{References}

1. Public Health Agency of Canada. Coronavirus Disease 2019 (COVID-19) Daily Epidemiological Update [Internet]. Government of Canada; 2020 May. https://www.canada.ca/content/dam/phac-aspc/documents/servi ces/diseases/2019-novel-coronavirus-infection/surv-covid19-epi-updateeng.pdf

2. Hsu AT, Lane N, Sinha SK, Dunning J, Dhuper M, Kahiel Z, et al. Impact of COVID-19 on residents of Canada's long-term care homes-ongoing challenges and policy responses [Internet]. International Long Term Care Policy Network; 2020. https://ltccovid.org/wp-content/uploads/2020/05/ LTCcovid-country-reports_Canada_Hsu-et-al_May-10-2020-2.pdf

3. Wang H, Li T, Barbarino P, Gauthier S, Brodaty H, Molinuevo JL, et al. Dementia care during COVID-19. Lancet. 2020;395(10231):1190-1.

4. Donelle L, Regan S, Kerr M, Zwarenstein M, Bauer M, Warner G, et al. Caring near and far by connecting community-based clients and family member/friend caregivers using passive remote monitoring: protocol for a pragmatic randomized controlled trial. JMIR Res Protoc. 2020;9(1):e15027

5. Folstein MF, Folstein SE, McHigh PR. "Mini-Mental State" A practical method for grading the cognitive state of patients for the clinician. J Psychiatr Res. 1975;2(12):189-98.
6. Sager MA, Rudberg MA, Jalaluddin M, Franke T, Inouye SK, Landefeld CS, et al. Hospital admission risk profile (HARP): Identifying older patients at risk for functional decline following acute medical illness and hospitalization. J Am Geriatr Soc. 1996;44(3):251-7.

7. Gale NK, Heath G, Cameron E, Rashid S, Redwood S. Using the framework method for the analysis of qualitative data in multi-disciplinary health research. BMC Med Res Methodol. 2013:13(1):117.

8. Fereday J, Muir-Cochrane E. Demonstrating rigor using thematic analysis: A hybrid approach of inductive and deductive coding and theme development. Int J Qual Methods. 2006;5(1):1-11.

9. Drury H, Ajmi S, Fernández de La Cruz L, Nordsletten AE, Mataix-Cols D. Caregiver burden, family accommodation, health, and well-being in relatives of individuals with hoarding disorder. J Affect Disord. 2014. 159(1):7-14.

10. Ringer T, Hazzan AA, Agarwal A, Mutsaers A, Papaioannou A. Relationship between family caregiver burden and physical frailty in older adults without dementia: a systematic review. Syst Rev. 2017;6(1):55.

11. Ontario Health and Wellness. COVID-19 action plan: long-term care homes | Ontario.ca [Internet]. 2020 [cited 2020 Jul 31]. https://www.ontar io.ca/page/covid-19-action-plan-long-term-care-homes

12. Dill JS, Cagle J. Caregiving in a patient's place of residence: turnover of direct care workers in home care and hospice agencies. J Aging Health. 2010;22(6):713-33.

13. Jeon $Y-H$, Simpson JM, Li Z, Cunich MM, Thomas TH, Chenoweth $L$, et al. Cluster randomized controlled trial of an aged care specific leadership and management program to improve work environment, staff turnover, and care quality. J Am Med Dir Assoc. 2015;16(7):629.e19-629.e28.

14. Keefe JM, Knight L, Martin-Matthews A, Légaré J. Key issues in human resource planning for home support workers in Canada. Work Read Mass. 2011;40(1):21-8.

15. Johnson S, Bacsu J, Abeykoon H, Mclntosh T, Jeffery B, Novik N. No place like home: a systematic review of home care for older adults in Canada. Can J. 2018;37(4):400-19.

16. Weeks LE, Shiner D, Stadnyk R, MacDonald D. What should we build when they are ready to come? Developing evidence-based housing for older adults. Sr Hous Care J. 2013;21(1):69-85.

\section{Publisher's Note}

Springer Nature remains neutral with regard to jurisdictional claims in published maps and institutional affiliations.
Ready to submit your research? Choose BMC and benefit from:

- fast, convenient online submission

- thorough peer review by experienced researchers in your field

- rapid publication on acceptance

- support for research data, including large and complex data types

- gold Open Access which fosters wider collaboration and increased citations

- maximum visibility for your research: over 100M website views per year

At $B M C$, research is always in progress.

Learn more biomedcentral.com/submissions 